\title{
New methodology for a person identification system
}

\author{
R BREMANANTH and A CHITRA \\ Department of Computer Science \& Engineering, PSG College of Technology, \\ Coimbatore 641 004, India \\ e-mail: bremresearch@gmail.com
}

MS received 28 April 2005; revised 16 January 2006

\begin{abstract}
Reliable person identification is a key factor for any safety measure. Unlike other biometrics such as the palm, retina, gait, face and fingerprints, the characteristic of the iris is stable in a person's lifetime. Iris patterns are chaotically distributed and well suited for recognizing persons throughout their lifetime with a single conscription. This paper proposes a new approach to person recognition based on iris patterns, which works with indoor outdoor conditions, spectacles contact lens wearing persons and diseased eyes. A challenge-response method is used for eye aliveness checking that puts off artificial sources from entering the iris database. The proposed algorithm can work with 84 statistical iris features that are extracted from an individual. Space and time complexity of the proposed approach is lesser than the existing methods. This algorithm has been implemented and results have been analysed on 2500 different iris patterns acquired in India under different real-time conditions. Experimental results illustrate that the proposed method has been easily espoused in elections, bank transactions and other security applications.
\end{abstract}

Keywords. Automatic recognition; biometrics; challenge-response test; iris segmentation; iris feature extraction; spatial domain.

\section{Introduction}

Biometrics proposes a new key that is user-friendly, intelligent and reliable for a person recognition system using human iris patterns. In fact, no two irises in the world are identical. Although identical twins have the same DNA making up their tissue structure, they have different iris patterns. Each iris is unique because a completely random process during fetal development forms it. Even a person's left and right eyes have completely different iris patterns. Hence, it can also be used as a kind of living password. A sample iris image is illustrated in figure 1. Figure 2 shows the biological features of the iris. Its complex pattern contains many distinguishing features such as arching ligaments, crypts, radial furrows, pigment frill, pupillary area, ciliary area, rings, corona, freckles and zigzag collarette. These spatial patterns in the iris are unique in nature. This technology works well in both recognition and confirmation phases. Fingerprint recognition (Jain et al 2000) is a very familiar system to identify a person; however this system causes more false positives than the iris recognition system, since fingerprints are often affected by external sources. Biometrics can be also deployed in 


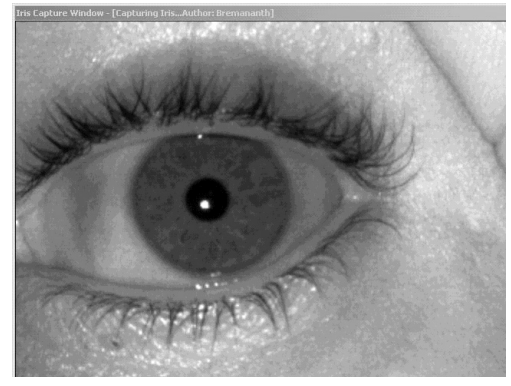

Figure 1. A sample eye image acquired by biometric camera.

the applications of cryptography to generate secret encryption key from the user biometric template. This may resolve the problems in digital rights managements (Uludag et al 2004).

The key issue of the pattern recognition problem is the relation between inter-class and intraclass variability. That is, classes can be efficiently differentiated only if the variability between features of a given class is less than the variability between other classes. In the iris recognition process, variability of iris intra-class features are less than the inter-class variability. In other biometrics recognition, such as fingerprints and face, intra-class variability is greater than inter-class variability. However, inter-class variability is limited because different classes hold the same basic set of features. The proposed iris recognition system initially acquires live eye images by testing the changes in diameter of pupil boundary from the same distance of capturing. This test is called the challenge-response test. Subsequently, iris segmentation, normalization, and enrichment phases are performed to make the iris patterns suitable for feature extraction process. Finally, the iris discriminator design phase classifies the irises. The overall organization of this paper is as follows: Section 2 summarizes the literature on the previous research. The algorithm of proposed iris recognition system is illustrated in $\S 3$. Experimental details and results are given in $\S 4$. Section 5 consists of our concluding remarks on this work.

\section{Review of literature}

In the process of iris recognition, it is essential to convert an acquired iris image into a suitable encoding feature that can be easily manipulated for iris verification and identification processes. In accordance with iris feature extraction, the iris recognition algorithm can be classified into four main approaches: Intensity variation analysis, phase-based analysis, zerocrossing analysis and texture analysis. Daugman (1993, 2004), Daugman \& Dowing (2001)

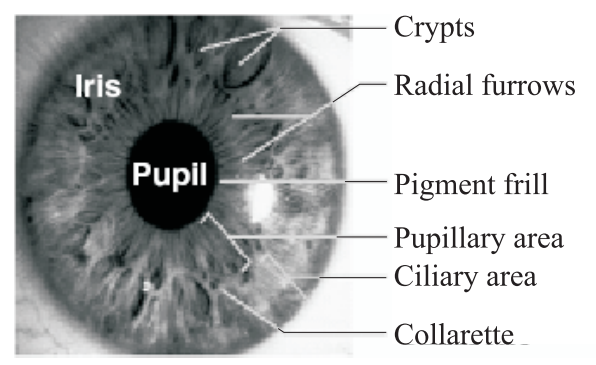

Figure 2. Biological features in the iris. 
proposed complex-valued multi-scale quadrature wavelets to extract phase structure information of the iris to generate a 2,048-bit (256 bytes) iris code. A pair of iris representations was compared based on Hamming distance. This approach belongs to the category of phase-based analysis. Ma et al $(2003,2004)$ suggested wavelets intensity signals for iris feature extraction, the similarity between a pair of iris was calculated using the exclusive OR operation. It is based on intensity variation analysis method. 2-D Haar wavelet transform was used to decompose an iris image into four levels and modified competitive neural network used for iris classification by Lim et al (2001). This method belongs to the texture analysis approach. Boles \& Boashash (1998) have implemented zero-crossing analysis of 1D wavelet transforms. It is used to locate various resolution levels of a concentric circle on an iris image. Iris features and iris matching are based on dissimilarity functions. In this approach, a new scheme for the iris recognition system is proposed, which includes eye liveness checking module to prevent artificial sources entering from the iris database. A compact scheme for iris feature extraction has been proposed by Gabor spatial transform. This algorithm provides translation, scale and rotation invariant (TSRI) recognition system based on texture analysis that is more suitable for any real-time security applications.

\section{Proposed iris recognition algorithm}

A block diagram of the proposed iris recognition system is shown in figure 3. The iris recognition system has seven modules: Eye image acquisition, challenge-response test (CRT), iris segmentation, iris normalization, iris enrichment, iris feature encoding and iris discriminator design.

\subsection{Eye image acquisition}

An important and complex step of iris recognition system is image acquisition. Especially for Indians, the iris is small in size and dark in colour. It is difficult to acquire clear images

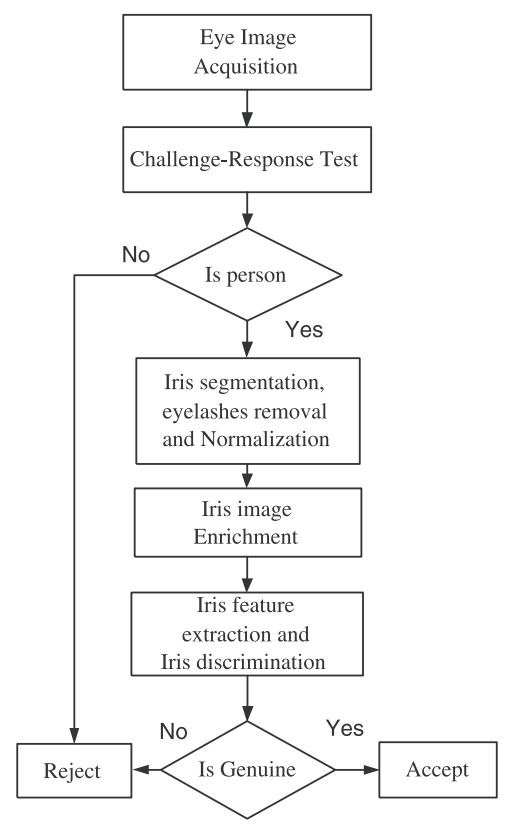

Figure 3. Block diagram of the proposed approach. 
using the standard CCD camera with ordinary lighting. In this module, a biometric camera is used to capture the user's eye images. It acquires images by passing NIR (near infrared) waves. The acquisition distance is normally between 19 and 36 inches and the average capturing time is 1.5 seconds. Due to distance or illumination changes, recognition process may produce different recognition rates. Perhaps, the same genuine candidate's iris features may slightly vary at sunlight and twilight. However, the system confines the Euclidean distance of the same candidate features to the iris discriminator phase. Research on iris image acquisition has been made in non-invasive imaging with just a few metres distance of separation. The image acquisition phase should consider three main aspects, namely, the lighting system, the positioning system, and the physical capturing system. Usually, in the enrolment phase iris images are captured without any eyewear that helps to encode the iris features accurately. However, the use of spectacles or contact lens by the user during verification does not affect the recognition process. The iris recognition system can work both in outdoor and indoor conditions without any hot spot of lighting intensities. However, unlike face, palm and fingerprints acquisition, irises are internal organs which are present inside the closed area of the eyelids; for this reason users must provide full cooperation for acquiring their eye images.

\subsection{Challenge-response test}

Biometric features may be counterfeited and criminally used. This is a crucial weakness of the biometric system. This module aims to ensure that an input image actually originates from a person instead of iris photographs, phony eyes, or other artificial sources. Daugman (2004) and Ma et al $(2003,2004)$ discussed these issues but did not present any specific scheme to surmount it. In our approach, a challenge-response test is suggested to overcome this problem.

The human iris is controlled by two muscles, namely the dilator and the sphincter, that allow the iris to adjust its size and control the amount of light entering the eye. If the amount of light entering the eye is increased, such as in bright light, then the sphincter muscle pulls towards the centre, the size of the pupil is constricted and less light is allowed to reach the retina. If the amount of light entering the eye is decreased, such as in a dark room or at night, then the dilator muscle pulls away from the centre, the size of the pupil enlarges and more light is allowed to reach the retina. By using these biological facts, our approach implements the challengeresponse test. This method verifies the response of the pupil diameter by varying illumination levels at the same distance from the eye. Diameter calculation is described in figure 4 .

The algorithm of this method is elaborated as follows:

Step 1: Capture the same person's eye images under different lighting levels (at least 3).

Step 2: Measure the pupil diameter from the captured eye images. If these values are divergent then the image is actually from a real source (human), otherwise artificial sources may have been used. The diameter of the pupil is calculated by satisfying (1). Equations (2) and (3) describe the challenge-response process.

$$
\begin{aligned}
& \left(x-x_{1}\right)\left(x-x_{2}\right)+\left(y-y_{1}\right)\left(y-y_{2}\right)=0, \\
& T_{d}=\sum_{i=0}^{n-1}\left|\Phi_{i}-\Phi_{i+1}\right|, \\
& C R T= \begin{cases}\text { True, } & \text { if } T_{d} \neq 0, \\
\text { False, } & \text { otherwise }\end{cases}
\end{aligned}
$$




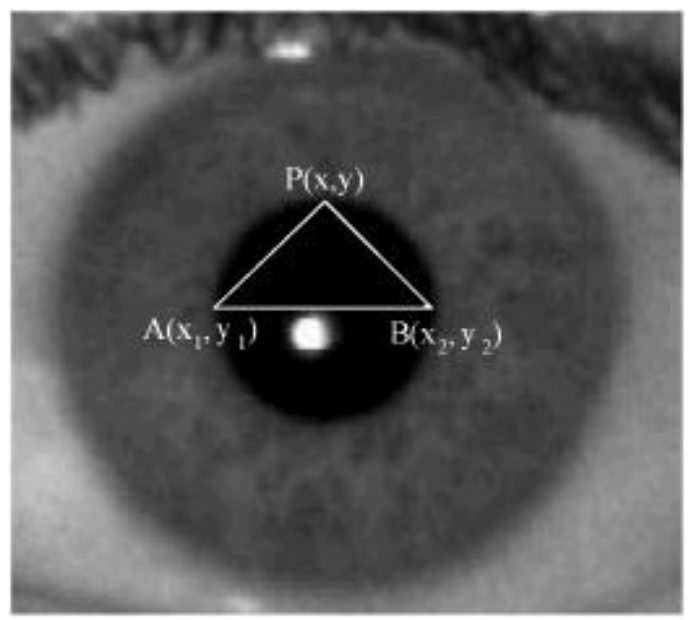

Figure 4. Measurement of diameter of the pupil boundary.

where $T_{d}$ is total diameter of the pupil in the capturing sequences, CRT is a challenge-response test parameter, $n$ is number of eye images, $\Phi_{i}$ and $\Phi_{i+1}$ are diameters of the pupil under different illuminations. This method assures that an input is coming from a real sequence and not from photographs or other artificial sources. The biometrics-capturing device needs to be capable of ensuring that they are inspecting genuine user features (as opposed to a photograph or recording) and that the output signal is not substituted. This is used to prevent replay attacks lifted from video-signals, for instance by connecting a video recorder to the frame-grabber.

\subsection{Iris segmentation}

Iris segmentation is the process to completely and efficiently eliminate pupil, eyelashes and other portions from the images that are not necessary for feature extraction and iris matching. Eliminating unnecessary data greatly saves time and boosts the efficiency of the system. In this module both the inner and outer boundaries of an iris can be considered as circles, but the two are usually not concentric. The steps involved in the segmentation process are summarized as follows.

Step 1: Input image is flipped $180^{\circ}$ vertically in order to avoid the detection of thick eyebrows as a pupil boundary. These types of images are often acquired in India because Indians have thick eyebrows, as is shown in figure 7a below.

Step 2: In order to increase the speed of the iris segmentation process, locate the iris by looking at the camera flash in the eye image. Canny operators are used for locating the camera flash. The operator finds the flash edges by looking for local maximum of the gradient of the image. Equations (4) and (5) illustrate this process:

$$
\begin{aligned}
& L g(x, y)=\left(G_{x}^{2}+G_{y}^{2}\right)^{1 / 2}, \\
& E d(x, y)=\tan ^{-1}\left(G_{y} / G_{x}\right),
\end{aligned}
$$

where $\operatorname{Lg}(x, y)$ is a local gradient function, $E d(x, y)$ is a edge direction, $G_{x}$ is the $x$-axis edge detector, and $G_{y}$ is the $y$-axis edge detector. The output of the camera flash detection is illustrated in figure 5 . 
(a)

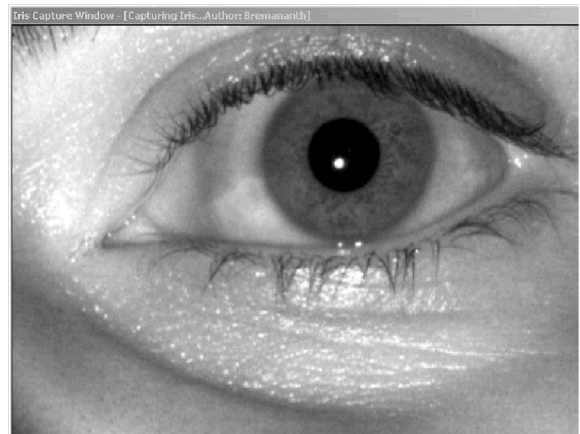

(b)

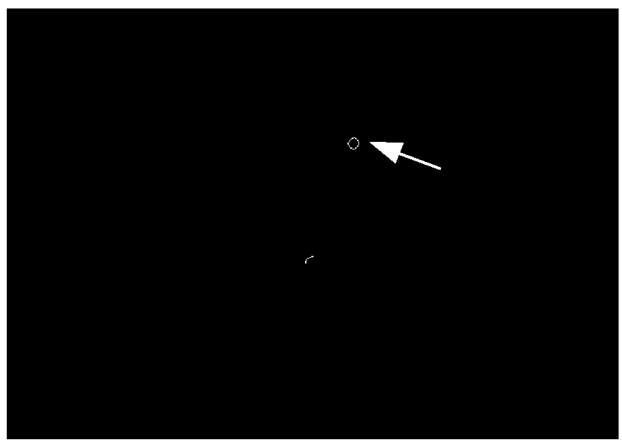

Figure 5. (a) Sample input eye image; (b) result of camera flash detection process.

Step 3: Cartesian co-ordinates of flash area are stored for detecting the inner boundary of the iris.

Step 3.1: Each coordinate in the flash area of the image is considered to be the centre of the circle and concentric circles are drawn by incrementing the radius with one until the threshold value $(\lambda)$ is reached, i.e., pupil threshold. Eight-way circular symmetry method is used to draw the circles.

Step 3.2: If the threshold value is satisfied, the radius $(\phi)$ and the location of centre pixel $(\rho)$ are stored.

Step 3.3: Repeat steps 3.1 and 3.2 until the maximum radius, $\phi$ is reached, i.e. repeat the process until the pupil's inner boundary is expected.

Step 4: In order to find the iris outer boundary, each pixel within the pupil is considered a centre and concentric circles are drawn using the 4-way circular symmetry method. All the pixel values in the boundary of the drawn circle are checked for the threshold value $(\Psi$, i.e. iris pixel threshold). The circle that contains the maximum number of pixel values that satisfy the threshold value $(\Psi)$ is the iris outer boundary. The processes of 8-way and 4-way circular symmetry method are illustrated in figures $6 \mathrm{a}$ and $6 \mathrm{~b}$ respectively. Results of iris segmentation process for various types of eye images are depicted in figure 7.

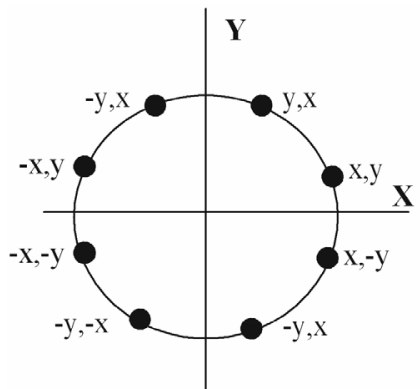

(a)

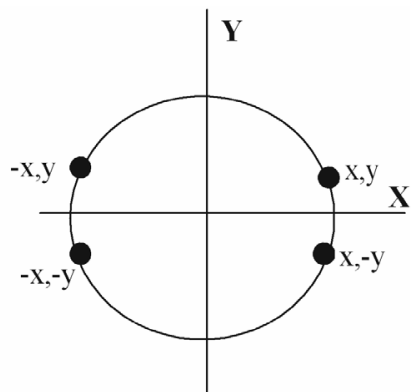

(b)
Figure 6. (a) Eight-way circular symmetry, (b) four-way circular symmetry. Calculation of one octant/quadrant yields the other seven octants/three quadrants. 


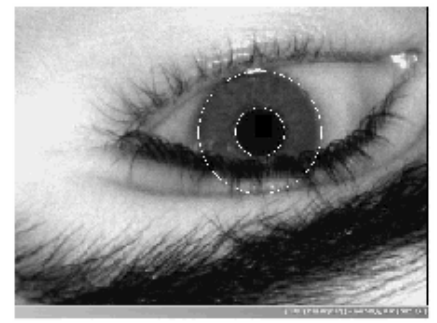

(a)

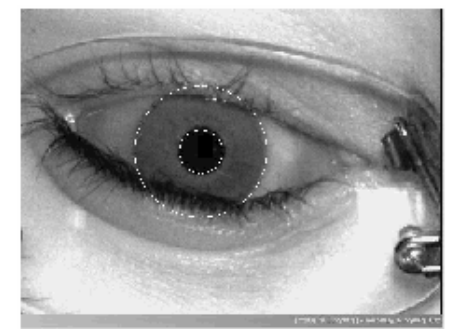

(b)

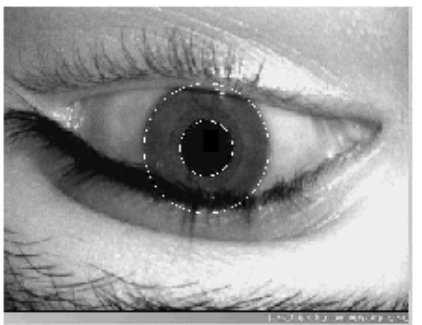

(c)

Figure 7. After detecting inner and outer boundaries of iris.

Eyelid/eyelashes occurring in the iris area should be eliminated properly because these portions are non-iris scrap in the iris portion that may produce misclassification in the irismatching process, and may change from session to session of capturing eye images. Ma et al (2004) reported $57.7 \%$ false non-matches due to the occlusion by eyelids/eyelashes. For this reason, the eyelid/eyelashes elimination component plays an important role in the recognition process. The iris image is contrasted by low-level pixel operation because the iris portion and the eyelashes/eyelids overlap each other and it is very difficult to eliminate these properly. The significant steps of elimination of eyelashes are as follows:

Step 1: Contrast is made by using a gain value $\beta$ ( $\beta$ value is 1.5 ), let $I$ be an iris pixel value. If $I$ is greater than the threshold $\eta$, then $I=I / \beta$, else if $(I * \beta)>\delta$ (i.e., $\delta$ is $0 \mathrm{xFFh}$ ), then $I=\delta$, else $I=I * \beta$.

Step 2: Contrasted iris pixel values are unpacked and checked to see whether these pixels values are expected threshold value $\omega$ or not (i.e. threshold value of eyelashes/eyelids is $0 \times 46 \mathrm{~h}$ ). These pixels are marked for detection (i.e., pixels are set to $0 \mathrm{xFFh}$ ).

Step 3: $\quad$ Marked pixels are properly collected and deleted from the iris portion. Figure 8a is after deletion of inner and outer boundaries of the iris portion. The elimination of eyelashes/eyelids is depicted in figure $8 \mathrm{~b}$.

\subsection{Iris image normalization}

As the iris is captured under different conditions like non-uniform illumination, eye blink, pupil radius change due to varying lighting etc., it is possible for the output of iris images to be in different sizes. This variations may affect the results of iris matching. To overcome this issue, iris image has been converted to standard size with width and height as 360 and 48 pixels respectively. Iris dilation or erosion process is also carried out to extend or squeeze iris strip size when fewer/higher amounts of data are obtained in the iris portion. This process is called iris normalization. In this process, the image is converted to standard rectangle strips using the radial scan method, which is used to collect all pixels in the iris portion and produce trapezoidal shape image as shown in figure 9b. Equation 6 describes scanning process of radial scan module.

$$
x=\oint x_{c}+r_{i} \cos ((\pi / 180) \theta), y=\oint y_{c}+r_{i} \sin ((\pi / 180) \theta)
$$



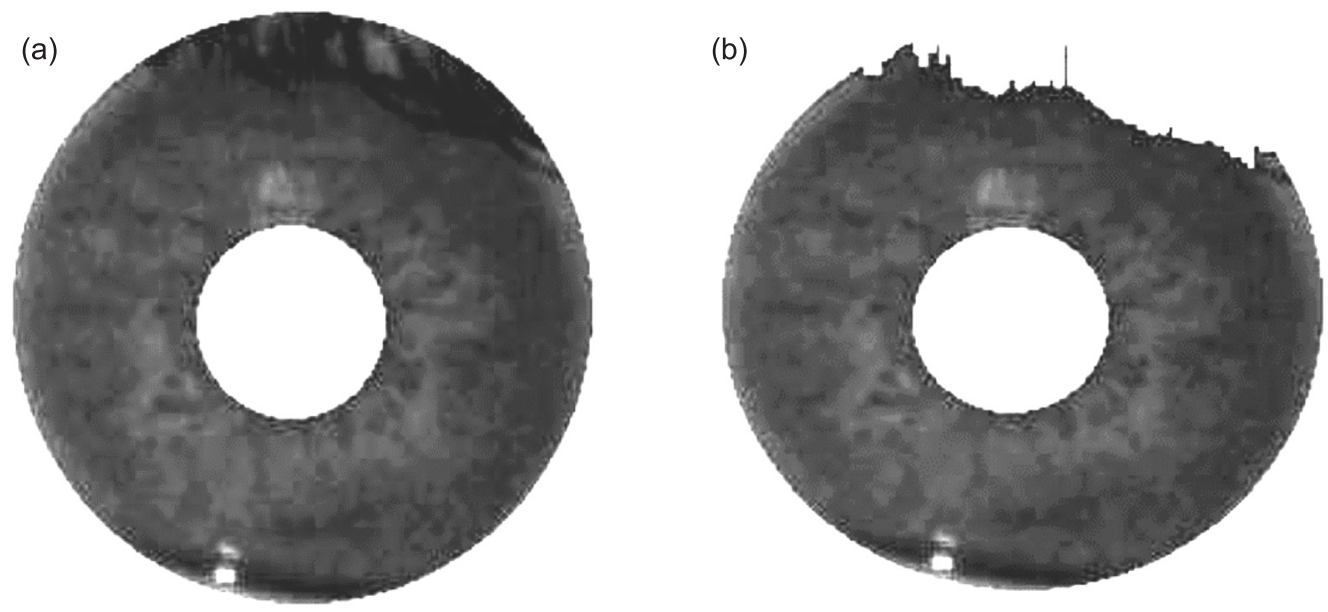

Figure 8. (a) After deleting inner and outer boundaries of the iris. (b) After elimination of the eyelashes/eyelids from the iris image.

where $x$ and $y$ are polar coordinates of the trapezoidal pattern, $x_{c}$ and $y_{c}$ are the centre of the circular iris pattern, $r_{i}$ is a radius of $i$ th scan. After forming trapezoidal pattern, the left triangular portion of the image is removed and mapped into the right portion of the iris image. After this process, dilation or erosion module can be performed to form a fixed size rectangle strip. Figure 9(a-d) describes various steps involved in the iris normalization process.

\subsection{Iris image enrichment}

Image enrichment process is based on spatial domain approach. The original iris image has low contrast and may have non-uniform illumination caused due to the irregular position of the light source. These problems may affect subsequent feature extraction and iris matching process. In order to obtain a well-distributed texture image, the iris image is enriched using local histogram equalization. Figure 10a shows the effect of applying histogram equalization process. Figures $10 \mathrm{~b}$ and show the histogram effect on the image enrichment process.

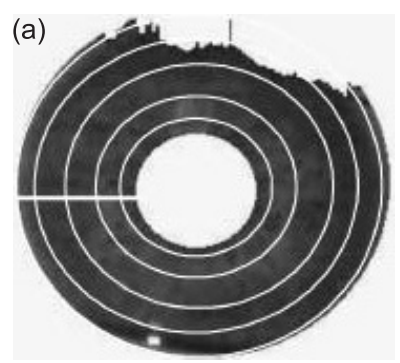

(b)

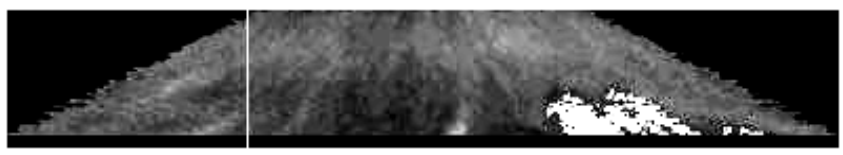

(c)

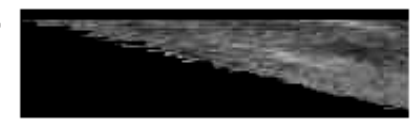

(d)

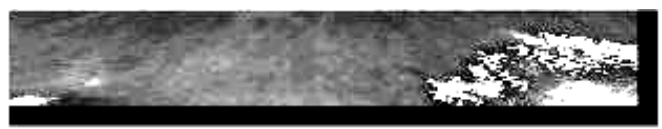

Figure 9. (a) Process of radial scan method, (b) output of radial process, (c) flopping operation for forming fixed size iris strip, (d) result of iris normalization process. 

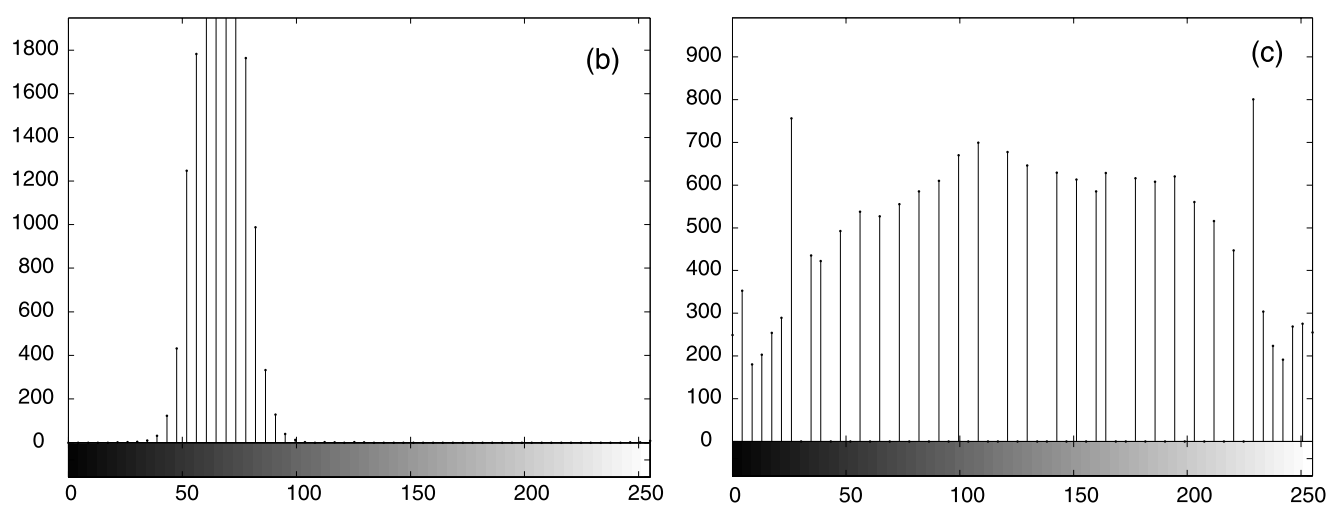

(a)
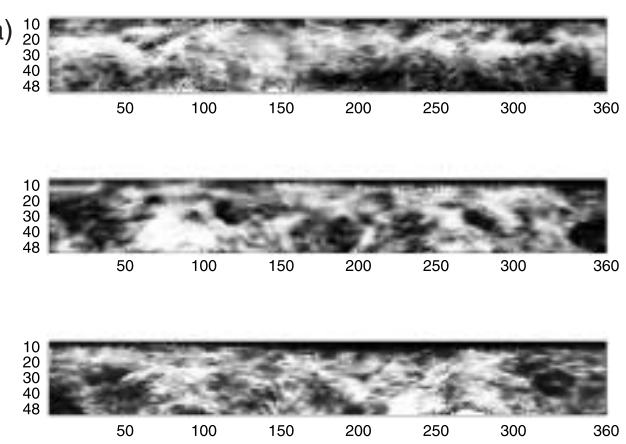

Figure 10. (a) Output of various iris patterns after enrichment process. (b) and (c) visualize the effect before and after applying the enrichment process.

\subsection{Iris feature encoding}

Iris feature encoding is a process to extract features from the iris image, which is subsequently used for the iris mapping process. Texture analysis is an important task for TSRI iris classification. In iris recognition process, iris images are captured in a noninvasive sense, i.e. no direct physical contact with the iris camera. It is possible to acquire images at different angles, positions, and scales due to distance (19-36 inches) variations. Hence, TSRI invariant feature extraction is required in the iris recognition system. In recent years, Gabor filters provide good performance in rotation-invariant texture classification. Hence, this system adopts Gabor filtering technique with isotropic 2D Gaussian for rotation-invariant iris feature encoding. Gabor elementary functions are Gaussian function modulated by oriented complex sinusoidal functions. Gabor frequency domain is defined as,

$$
\begin{aligned}
& G(x, y)=g(x, y) \exp (-2 \pi j(u \cdot x+v \cdot y)) \\
& g(x, y)=\frac{1}{2 \pi \sigma^{2}} \exp \left\{-\left[\frac{x^{2}+y^{2}}{2 \sigma^{2}}\right]\right\}, j=\sqrt{-1} .
\end{aligned}
$$

The complex function $G(x, y)$ can be split into two parts, even and odd filters, $G_{e}(x, y)$ and $G_{o}(x, y)$, which are also known as symmetric and anti-symmetric filters respectively. The spatial Gabor filter is described as

$$
\begin{aligned}
& G_{e}(x, y)=g(x, y) \cos (2 \pi f(x \cos \theta+y \sin \theta)), \\
& G_{o}(x, y)=g(x, y) \sin (2 \pi f(x \cos \theta+y \sin \theta)),
\end{aligned}
$$




\begin{tabular}{|c|c|c|}
\hline 0.000000 & 0.000837 & 0.000000 \\
\hline 0.000811 & 2.547771 & 0.000811 \\
\hline 0.000000 & 0.000837 & 0.000000 \\
\hline
\end{tabular}

Figure 11. Illustration of typical Gabor kernel with $\theta=90^{\circ}$ and $f=64$.

where $G(x, y)$ is the Gabor filters kernel, $g(x, y)$ is an isotropic 2D Gaussian function, $f$ is the centre frequency, $\theta=\arctan (u / v)$ is the orientation.

An iris strip of fixed size $(360 \times 48)$ is taken for the feature extraction process. Selection of Gabor filter parameters plays an important role in the rotation-invariant pattern classification problem. Depending upon the application, the system can choose parameters such as radial frequency of the sinusoid and orientation. Performance of the Gabor filter depends on choosing its parameters $(\theta, f)$ because it produces widely varied responses in different types of parameter settings. The central frequencies of Gabor filters used in the experiment range from 2 to 64 degrees. For each central frequency, orientation ranges as $\theta=0^{\circ}-180^{\circ}$. Totally, 42 Gabor kernels are generated for feature encoding. The rectangular strip of iris pattern is divided into 2 sub images; each has a $180 \times 48$ strip. Forty-two kernels are applied to 2 subimages. A typical Gabor kernel is illustrated in figure 11. In order to minimize the computation complexity of the iris mapping process, the iris strip is convoluted by polynomial multiplication as,

$$
\operatorname{Iris} F(x, y)=\sum_{k=-1}^{1} \sum_{j=-1}^{1} G(j, k) I(x-j, y-k),
$$

where Iris $F(x, y)$ is a result of convolution process which contains iris coefficients. $G(j, k)$ is a Gabor kernel and $I(x, y)$ is a fixed size iris strip.

The mean value of each frequency and orientation of the iris strip has calculated from the result of convolution process as,

$$
\bar{x}=\frac{1}{N \times M} \sum_{i=1}^{N} \sum_{j=1}^{M} \operatorname{Iris} F\left(x_{i}, y_{j}\right),
$$

where $N, M$ is the size of the iris coefficients and $\bar{x}$ is an iris feature code. In the proposed approach, 84 iris features are extracted from the given iris pattern. These features represent pigment changes in the iris patterns. As we do Gabor kernel convolution operation, these pigment changes are produced as a set of mean values. By changing the kernel parameters the filters respond to diverse mean values for each iris pigment pattern in a rotation-invariant manner. These iris codes are stored in an encrypted flat file to determine whether two candidate irises are from the same class or not. Moreover, this system selects iris features from the iris patterns after convolving by Gabor kernels. Thus, these features are aptly selected by error difference between applying one set of parameters to another. Therefore, to a large extent, we can use Gabor texture features for classifying iris patterns. In addition, to minimize the error rate of the intra-class variability of irises, the system needs an optimum method to estimate a minimum rate. We estimate the error rate for all possible combinations of the two parameters 
and then find the optimal values that minimize the error rates. Thus, the system can achieve good response in rotation-invariant iris pattern analysis by computing error differences of Gabor filter responses.

\subsection{Iris discriminator design}

In order to distinguish a candidate's iris class, an iris feature is represented as a 1D feature vector of size 84 per candidate. Feature set $F S_{i}=\left\{f_{1}, f_{2}, \ldots \ldots f_{84}\right\}$ is used for the recognition process. These sensitive features are the mean value of resultant Gabor kernel convolution operation. During the testing, standard deviation values of known iris patterns are calculated from the set of known mean features. In this proposed approach, the Euclidean norm distance measurement (ENDM) discriminator is employed to separate intra- and interclass variations of iris patterns. To authenticate a genuine user, iris feature sets are treated as known sets that are already stored in the encrypted file and verification candidates' iris features are represented as unknown sets. The same candidate's iris feature codes may vary due to external noises, lighting, obscuring by eyelashes etc. During any instant when no unknown set is present, the probability density for a known set is normal, that is, $p\left(x \mid \omega_{1}\right) \sim K\left(\mu_{1}, \sigma^{2}\right)$ when the unknown set is present, the density is $p\left(x \mid \omega_{2}\right) \sim K\left(\mu_{2}, \sigma^{2}\right)$. Any decision threshold $X^{*}$ will determine the probability of a hit and of a false alarm. As per signal detection theory, ENDM is described as,

$$
\begin{aligned}
\operatorname{ENDM}\left(F S_{i}\right)= & \sum_{i=1}^{n}\left(F S_{i} \mu_{2}-F S_{i} \mu_{1}\right)^{2} / F S \sigma_{i}^{2} \\
F S \sigma_{i}= & {\left[\left[N M \sum_{i=1}^{N} \sum_{j=1}^{M} I\left(x_{i}, y_{j}\right)^{2}-\left(\sum_{i=1}^{N} \sum_{j=1}^{M} I\left(x_{i}, y_{j}\right)\right)^{2}\right]\right.} \\
& \div[N(N-1) M(M-1)]]^{1 / 2},
\end{aligned}
$$

where $F S_{i} \mu_{2}$ is an unknown iris feature code, $F S_{i} \mu_{1}$ is a known iris feature code and $F S \sigma_{i}$ is the standard deviation of the known feature code. In the proposed approach, the threshold value of the decision boundary of ENDM ranges from 0.0 to 0.4 . If the boundary is raised up to 0.4 , the probability of a hit is present, i.e., a genuine candidate has been recognized, otherwise it is a false alarm.

\section{Experimental results}

Implementation has been done in Java, result analysis are studied in MatLab6. 0 and time efficiency is based on observations made on a Pentium IV $2.4 \mathrm{GHz}$ machine with 256 MB RAM. Capturing eye images in different situations provides more challenges to this approach. Experiments were done with different eye images under diverse conditions such as with normal eyes, out of doors, wearing contact lens spectacles, and diseased (tumours, tear, iridocyclities) irises. Some possible eye images are shown in figure 12. The database of iris images has 2500 images captured from 500 different candidates as illustrated in table 1. These feature sets are referred to as $f s_{1}, f s_{2} \ldots f s_{2500}$. The iris database has $95 \%$ images acquired from Indian candidates and 5\% from foreigners. This iris database is considered to be the biggest in India as per the reports analysed. 


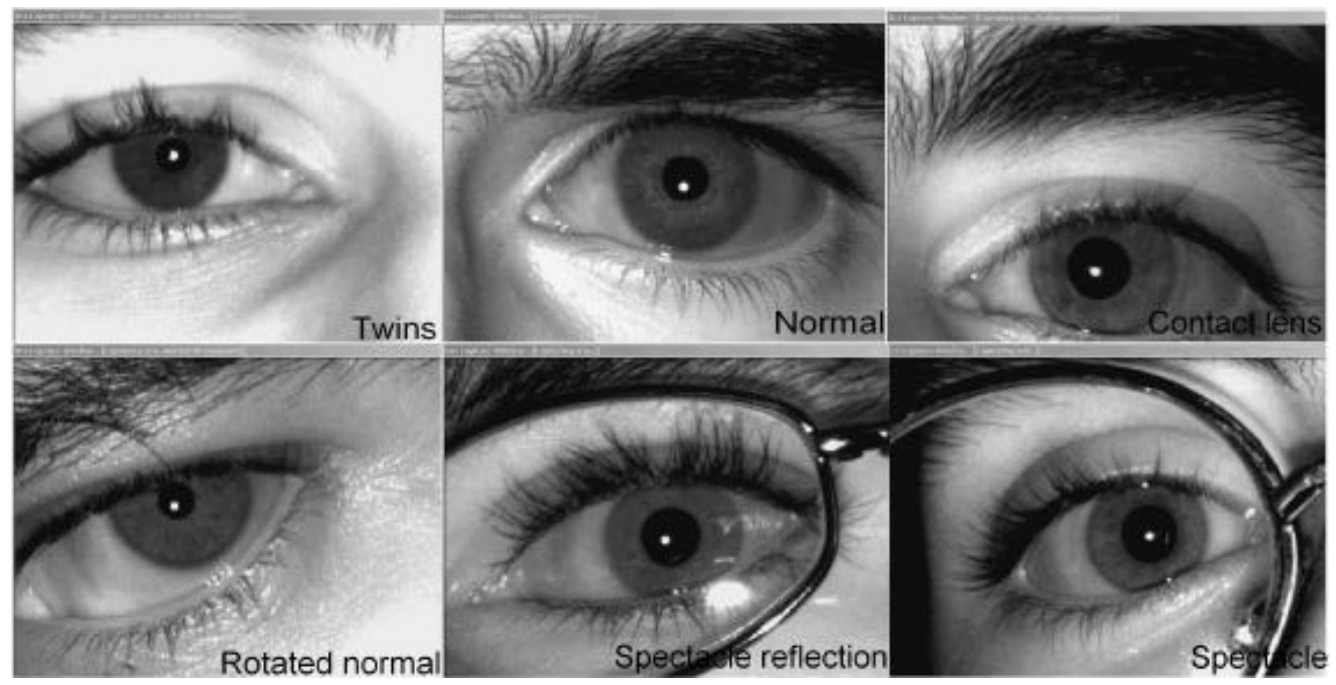

Figure 12. Sample eye images acquired under different conditions.

The process by which a user's biometric data is initially acquired, validated, processed and stored in the form of a template for ongoing use in a biometric system is called enrolment. Quality enrolment is a critical factor in the long-term accuracy of biometric systems. This approach performs various modules like challenge-response test and image preprocessing, ensuring quality enrolment of the system.

Although iris patterns are unique in nature, a user could position the same eye on a biometric device for years and never generate identical iris templates. This is due to the iris patterns change in positioning, distance, illumination conditions, and other factors such as closed eyelashes or eyelids that affect biometric acquisition system. Capturing eye images with costly biometric camera may solve this problem but this is suitable only for large-scale applications. Also, for iris recognition, the user must extend full cooperation in imaging to acquire welldistributed iris pigments. The process of comparing iris features to determine their degree of similarity or correlation is called iris matching. This algorithm was tested in two different phases of operations, namely, recognition and confirmation phases. In both phases, the system

Table 1. Iris database.

\begin{tabular}{|c|c|c|c|c|c|}
\hline Candidates & Group/gender & Age & & Criterion & Acquisition \\
\hline \multirow[t]{3}{*}{ Indian } & Female & $\begin{array}{l}10 \geq \text { and } \leq 20 \\
21>\text { and }<60\end{array}$ & $\begin{array}{l}20 \% \\
25 \%\end{array}$ & \multirow{4}{*}{$\begin{array}{l}\text { Normal, } \\
\text { wearing } \\
\text { contact lens } \\
\text { spectacles, } \\
\text { diseased, } \\
\text { indoors and } \\
\text { outdoors }\end{array}$} & \multirow{4}{*}{$\begin{array}{l}\text { Two months } \\
\text { Interval } \\
\text { between two } \\
\text { capturing } \\
\text { stages }\end{array}$} \\
\hline & Male & $10 \geq$ and $\leq 20$ & $20 \%$ & & \\
\hline & $\begin{array}{l}\text { Genetically } \\
\text { related members } \\
\text { (family members) }\end{array}$ & $21 \geq$ and $\leq 60$ & $5 \%$ & & \\
\hline Foreigner & Both male and female & $21 \geq$ and $\leq 40$ & $5 \%$ & & \\
\hline
\end{tabular}




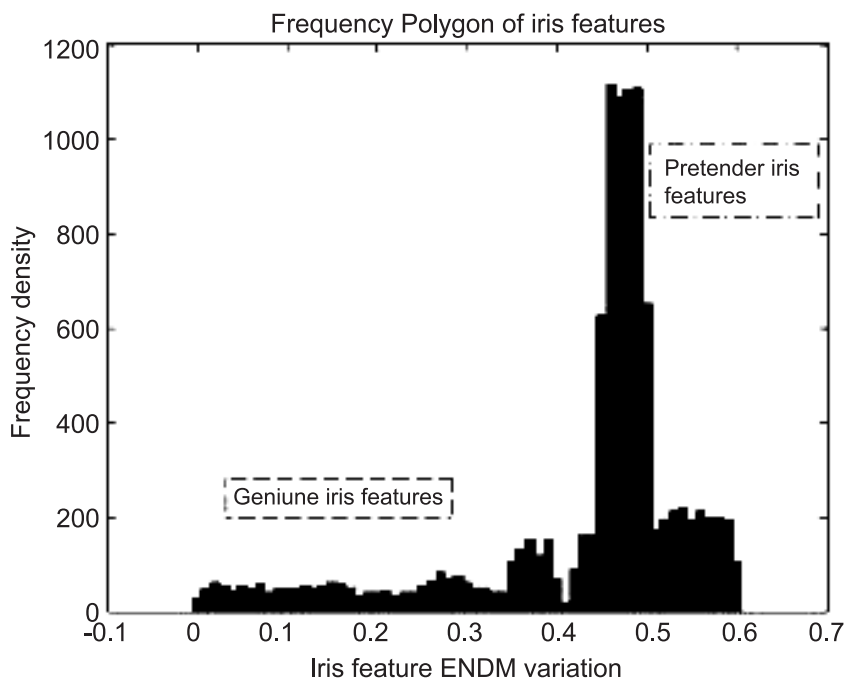

Figure 13. Distribution of iris features in ENDM.

identifies genuine subjects by checking ENDM. This is due to various artifacts occurring in iris patterns during verification.

\subsection{Iris recognition phase}

In this phase, the test iris feature set was compared against the remaining enrolled iris feature sets. That is, $N(N-1)$ comparisons were performed, is 6247500 possible verifications in the iris database. The threshold value of ENDM was set as 0.4. The distribution of iris features was measured and is shown in figure 13. Mean and standard deviations of genuine iris features were 0.232915 and 0.021230 respectively. The degree-of-freedom was $396 \cdot 40\left(\mu(1-\mu) / \sigma^{2}\right)$. The "pretender" iris features mean was 0.491319 and standard deviation was 0.040131 . The discriminability factor was 8.049 . The proposed method provides genuine acceptance rate (GAR) of $99.96 \%$ and false acceptance rate (FAR) of $0.04 \%$. GAR and FAR of recognition phase are described by

$$
\mathrm{GAR}=1-\mathrm{FAR}, \mathrm{FAR}=\mathrm{FA} / N
$$

where FA is number of false acceptances and $N$ is the total number of iris feature sets. FAR is also known as false match rate, and describes the number of times some one's iris is inaccurately matched with another's.

\subsection{Iris confirmation phase}

In this phase, test iris feature set is compared against an already existing enrolled iris feature sets with specific key. The key may be a date of birth or an access number of the candidates. It provides one to one match against enrolled iris feature sets. This phase uses the threshold values as maintained in the recognition phase. Matching rate (MR) of the proposed method was $99.96 \%$ and false rejection rate (FRR) was $0.04 \%$. MR and FRR of confirmation phase is described as,

$$
\mathrm{MR}=1-\mathrm{FRR}, \mathrm{FRR}=\mathrm{FR} / N,
$$


where FR is the number of episodes of false rejection and $N$ is the total number of iris feature sets.

FRR is also known as false non-match rate (FNMR), which gives the number of times a genuine candidate's iris is rejected instead of begin accepted.

In both the phases, left and right irises may be used for recognition, which provides more security in application domains. This is just like a double padlock facility. However, as per the confirmation phase, this is not a time-consuming process, but in the recognition process, it requires more time to validate both the patterns.

\subsection{Failure-to-enrol}

In this result analysis phase, the probability of failure-to-enrol (FTE) rate has been studied in accordance with the challenge response test and image preprocessing modules. It represents the probabilities that given users are unable to enrol in the iris database. Interestingly, adjusting the system setting to reduce FTE rates can also affect FNMR. In the experiments, FTE was $0.08 \%$ because of the efficiency of the image preprocessing module. The CRT module prevents illegal entries being entered into the iris database.

\subsection{Ability-to-verify rate}

Ability-to-verify (ATVR) is a combination of FTE and FNMR that indicates the overall percentage of candidates who are capable of authenticating on a timing basis. ATVR as below is $99.88 \%$,

$$
\text { ATVR }=(1-\text { FTE })(1-\text { FNMR }),
$$

where AVTR is ability-to-verify rate, FTE is false-to-enrolrate, FNMR is false-non-match rate of the system. Cost, security and convenience of the biometric system depends on the acceptable FMR, ATVR.

\subsection{Overall performance}

The proposed system has been assessed by various metrics such as MR, GAR, FNMR, FMR, FTE, and AVTR. In addition to these metrics, EER (equal error rate) is also calculated to

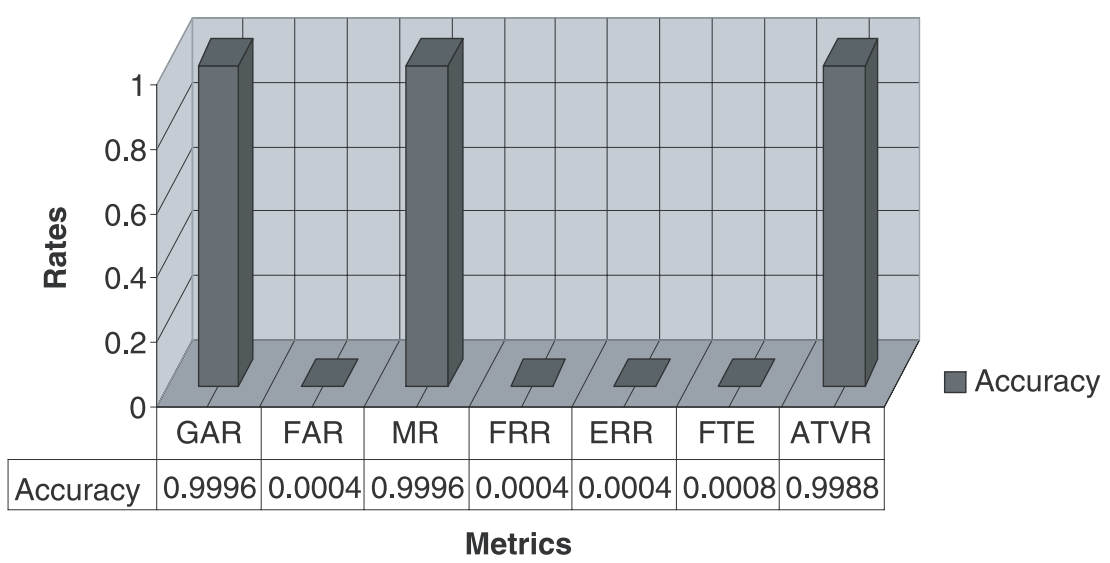

Figure 14. Overall performance of the proposed iris recognition system. 
Table 2. Samples data for sensitivity and specificity testing.

\begin{tabular}{llclcc}
\hline Enrolled iris feature set & \multicolumn{2}{c}{ Non-enrolled iris feature set } & \multicolumn{2}{c}{ Total } \\
\hline Positive & True positive (GAR) & 2499 & False positive (FAR) & 1 & 2500 \\
Negative & False negative (FRR) & 1 & True negative (TR-true rejection) & 1249 & 1250 \\
\hline
\end{tabular}

evaluate crossover rate of the proposed system. If FNMR is equal to the FMR, the system is called as EER. EER of the proposed system was $0.04 \%$ of rate. Figure 14 depicts the accuracy of various metrics of the proposed approach.

In all pattern recognition problems, the key issue is the relation between inter-class (different candidates) and intra-class (same candidate) variability. A candidate can be constantly classified only if the variability among different eye images of the same candidate's features is less than the variability between different candidates. The proposed approach uses texture TSRI pattern recognition system by using Gabor filters. It provides both global and local variations of iris texture features and increases the efficiency of the system. Successive capturing of iris images may generate slightly different iris feature codes. Moreover, due to eyewear and diseased eyes, Gabor texture features could change to some extent. Thus its ENDM was confined between 0.0 and 0.4 to confirm genuine irises. To avoid these artifacts, we propose a method for removing eyewear from the acquired eye images or check the status of the images before feature encoding or enhance the eye images. This approach uses the iris image enrichment phase. For the experiment, iris images of the same candidate were captured once every one-hour and once in two months to evaluate their intra-class and inter-class variability.

4.5a Receiver operating characteristics curve (ROC): The performance tradeoff between sensitivity (true positive rate) and specificity (true negative rate) of the system is illustrated by the receiver operating characterstics curve (ROC). Sensitivity is the probability that a test will be positive when the candidate iris feature set is present in the database. The specificity is a probability that a test will be negative when the candidate iris feature set is not present in the database. FAR is the probability of accepting a cheat or impersonator as an authorized candidate and GAR is the probability of accepting an authorized candidate. To test the ROC of the proposed approach, 250 non-enrolled candidates iris images have been acquired in five different sessions, hence, an additional 1250 iris images (non-enrolled) have been tested against the already stored 2500 (enrolled) images. The sensitivity and specificity of the proposed system is illustrated in tables 2 and 3. The following equations depict the process of ROC.

$$
\begin{array}{rlrl}
S n & =\mathrm{GAR} /(\mathrm{GAR}+\mathrm{FRR}), & & S p=\mathrm{TR} /(\mathrm{FAR}+\mathrm{TR}) \\
\mathrm{PLR} & =S n /(1-S p), & \mathrm{NLR}=(1-S n) / S p, \\
\mathrm{PRV} & =\mathrm{GAR} /(\mathrm{GAR}+\mathrm{FAR}), & \mathrm{NPV}=\mathrm{TR} /(\mathrm{FRR}+\mathrm{TR}) .
\end{array}
$$

Table 3. Result of sensitivity and specificity rates.

\begin{tabular}{lclc}
\hline Sensitivity & 0.9996 & Specificity & 0.9992 \\
Positive likelihood ratio & {$[0.9996: 0.0008]$} & Negative likelihood ratio & {$[0 \cdot 0004: 0.9992]$} \\
Positive predictive rate & 0.9996 & Negative predictive rate & 0.9992 \\
\hline
\end{tabular}




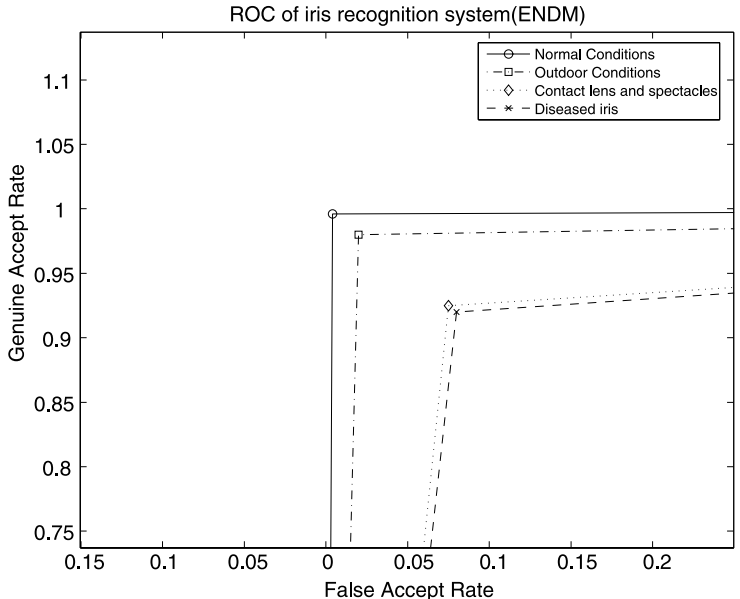

Figure 15. Illustration of ROC measurements in iris recognition.

where $S n$ is sensitivity rate, $S p$ is specificity rate, TR is true rejection rate, PLR is positive likelihood rate, NLR is negative likelihood rate, PRV is positive predictive value, and NPV is negative predictive value.

Positive likelihood rate is the rate between the presence and absence of the probability of positive test result given by enrolled iris feature sets. Positive predictive rate is the probability that the iris feature set is present when the test is positive. Negative predictive value is a probability that the iris feature set is not present when test is the negative. The tradeoff between GAR and FAR has been tested under four different conditions such as normal and outdoor conditions, wearing contact lens/spectacles and with diseased irises. Figure 15 illustrates the result of ROC analysis. It shows that the proposed approach is reliable for real-time applications.

4.5b Space and time complexity: Storage requirement for iris matching is considerably reduced in this approach. Totally 84 features were used to identify different persons, i.e., $84 \times 16$ bits $=168$ bytes were desirable per iris. Totally, 2500 eye images features were extracted and stored in $410 \cdot 15 \mathrm{~KB}$ of an encrypted file. Execution times of the recognition processes were measured and are listed in table 4.

Table 4. Time complexity of iris recognition processes.

\begin{tabular}{ll}
\hline Significant steps in iris recognition & Time (s) \\
\hline Detect flash & $0 \cdot 19$ \\
Segmentation & $0 \cdot 231$ \\
Normalization & $0 \cdot 276$ \\
Enrichment & $0 \cdot 1$ \\
Feature extraction & $0 \cdot 203$ \\
Confirmation & $0 \cdot 122$ \\
\hline
\end{tabular}


Table 5. Comparison of features, space and time complexity.

\begin{tabular}{lccc}
\hline Approaches & $\begin{array}{c}\text { Number of feature } \\
\text { components }\end{array}$ & Feature extraction time (s) & Iris confirmation (s) \\
\hline Ma et al (2004) & 660 & $0 \cdot 244$ & $0 \cdot 652$ \\
Ma et al (2003) & 1536 & $0 \cdot 440$ & $0 \cdot 131$ \\
Daugman (2004) & 2048 & $0 \cdot 723$ & $0 \cdot 004$ \\
Lim et al (2001) & 87 & $0 \cdot 824$ & $0 \cdot 232$ \\
Proposed system & 84 & $0 \cdot 203$ & $0 \cdot 122$ \\
\hline
\end{tabular}

\subsection{Comparison with existing approaches}

The proposed approach has been compared with existing approaches in accordance with feature component, space and time complexities. Each system has its own merits/demerits and different processing modules. Table 5 shows the comparison report. The proposed approach outperforms the other methods in challenge-response test, eyelashes/eyelid removal, compact features, and time/space requirements. The experimental results were illustrated that this system can easily be adopted for any real-time applications.

\section{Conclusion}

This article proposes a new algorithm for identification of persons using human iris patterns that works under indoor and outdoor conditions, with eyewear or diseased eyes. The challengeresponse test is used for checking eye aliveness, which ensures detection of counterfeit irises, and is compared with data enrolled from the iris-encrypted file. This algorithm locates irises more efficiently than existing approaches. Moreover, it properly deletes eyelashes/eyelids that occur in the iris portion. This system aims to perform iris pattern analysis in the TSRI mode. Gabor texture filters are employed for feature extraction that encodes iris features in the rotation invariant approach. Iris-matching process is performed by Euclidean norm distance measurement. Compared with existing methods, the space complexity of this method is considerably reduced. The results of the analysis shown that this proposed approach is suitable for any real-time applications such as e-voting, employee management, terrorist identification, passport verification and other applications. It opens a new avenue in iris recognition research work. In further development, this system can be improved to identify a person at a few metres distance and a new approach will be suggested to delete eyewear from acquired images and minimize artifacts occurring in the eye area.

\section{References}

Boles W W, Boashash B 1998 A human identification technique using images of the iris and wavelet transform. IEEE Trans. Signal Process. 46: 1185-1188

Chitra A, Bremananth R 2003 Secure PID using iris pattern based on circular symmetric and Gabor filters. Proc. of Int. Conf. Advanced Computing and Communication (ADCOM), pp 36

Chitra A, Bremananth R 2004 Efficient identification based on human iris patterns. Proc. of Fourth Indian Conf. on Computer Vision, Graphics and Image Processing (ICVGIP 2004), pp 177-183

Daugman J 1993 High confidence visual recognition of persons by a test of statistical independence. IEEE Trans. Pattern Anal. Machine Intell. 15: 1148-1161 
Daugman J 2004 How iris recognition works. IEEE Trans. Circuits Syst. Video Technol. 14: 21-30 Daugman J, Dowing C 2001 Epigenetic randomness, complexity and singularity of human iris patterns. Proc. R. Soc. B268: 1737-1740

Grigorescu S E, Petkov N, Kruizinga P 2002 Comparison of texture features based on Gabor filters. IEEE Trans. Image Process. 11: 1160-1167

Jain A K, Prabhakar S, Hong L, Pankathi S 2000 Filter-bank based fingerprint matching. IEEE Trans. Image Process. 9: 846-859

Lim S, Lee K, Byeon O, Kim T 2001 Efficient iris recognition through improvement of feature vector and classifier. ETRI J. 23: 61-70

Ma L, Tan T, Wang Y, Zhang D 2004 Efficient iris recognition by characterizing key local variations. IEEE Trans. Image Process. 13: 739-750

Ma L, Tan T, Wang Y, Zhang D 2003 Personal identification based on iris texture analysis. IEEE Trans. Pattern Anal. Machine Intell. 25: 1519-1533

Uludag U, Pankanti S, Prabhakar S, Jain A K 2004 Biometric cryptosystems: Issues and challenges. Proc. IEEE 92: 948-960 